$01,05,11$

\title{
Влияние гидрирования на магнитострикцию и магнитокалорический эффект в монокристалле гадолиния
}

\author{
(C) И.С. Терешина ${ }^{1}$, Г.А. Политова ${ }^{2,3}$, В.А. Четырбоцкий ${ }^{1}$, Е.А. Терешина-Хитрова ${ }^{4,5}$, \\ М.А. Пауков ${ }^{4,6}$, А.В. Андреев ${ }^{5}$ \\ ${ }^{1}$ Московский государственный университет им. М.В. Ломоносова, \\ Москва, Россия \\ ${ }^{2}$ Институт металлургии и материаловедения им. А.А. Байкова РАН, \\ Москва, Россия \\ ${ }^{3}$ Санкт-Петербургский политехнический университет Петра Великого, \\ Санкт-Петербург, Россия \\ ${ }^{4}$ Карлов университет, \\ Прага, Чешская Республика \\ ${ }^{5}$ Институт фоизики ЧАН, \\ Прага, Чешская Республика \\ ${ }^{6}$ Балтийский федеральный университет им. Иммануила Канта, \\ Калининград, Россия \\ E-mail: irina_tereshina@mail.ru
}

(Поступила в Редакцию 10 сентября 2018 г.)

Монокристалл гадолиния, полученный по методу Чохральского, был подвергнут наводороживанию до состава $\mathrm{GdH}_{0.15}$, что соответствует твердому раствору металл-водород ( $\alpha$-фаза). Для исходного и гидрированного образцов были измерены магнитострикция и магнитокалорический эффект. Установлено, что атомы водорода в гексагональной решетке гадолиния способны оказывать влияние на величину и знак магнитострикционных констант, а также приводить к анизотропии магнитокалорического эффекта. Обсуждены основные механизмы, ответственные за наблюдаемые эффекты.

Работа выполнена при поддержке РФФИ, проект № 16-03-00612.

DOI: 10.21883/FTT.2019.02.47118.253

\section{1. Введение}

Редкоземельные металлы (РЗМ) вызывают значительный интерес исследователей на протяжении достаточно большого времени [1], поскольку изучение свойств этих металлов имеет не только важное научное, но и прикладное значение. Редкоземельные металлы, их сплавы и соединения широко используются во многих областях современного производства, а именно в атомной энергетике и металлургии, нефтеперерабатывающей, стекольной, керамической и оптической промышленности, электронике и медицине [2-4]. Среди всего ряда РЗМ особое внимание исследователи уделяют гадолинию [5-7]. Он является функциональным магнитным материалом (к примеру, рабочим телом магнитного рефрижератора) с температурой Кюри вблизи комнатной $\left(T_{\mathrm{C}}=293 \mathrm{~K}\right)$. Ниже $T_{\mathrm{C}}$ гадолиний (единственный среди магнитных Р3М) имеет ферромагнитное упорядочение, которое сохраняется при охлаждении вплоть до самых низких температур. При $T=220 \mathrm{~K} \mathrm{Gd}$ демонстрирует спинпереориентационный переход (СПП) типа „ось легкого намагничивания" - ,легкий конус“.

В рефрижераторах гадолиний работает в водородсодержащих средах. Именно поэтому исследование влияния гидрирования на магнитные свойства гадолиния крайне важно. Ранее, нами уже было изучено влияние водорода и азота на температуру Кюри, магнитокалорический эффект (МКЭ) в области $T_{\mathrm{C}}$, а также на механические свойства для структурно-неоднородных образцов $\mathrm{Gd}$, полученных после процедуры дистилляции [8-12]. Цель настоящей работы - изучить аномалии магнитострикции и магнитокалорического эффекта в монокристалле $\mathrm{Gd}$ до и после гидрирования вблизи двух магнитных фазовых переходов: „порядок-порядок“ (в области СПП) и „Порядок-беспорядок“ (в области температуры Кюри).

\section{2. Получение образцов и методика эксперимента}

Монокристалл Gd выращивали в атмосфере аргона методом Чохральского, используя для этого дуговую печь, медный водоохлаждаемый тигль и вольфрамовый стержень (в качестве элемента затравки). Подтверждение монокристаллического состояния полученного образца, а также ориентация его вдоль основных кристаллографических направлений проводилась с помощью метода Лауэ. Как результат, были вырезаны пластины с размерами $8 \times 4 \times 2 \mathrm{~mm}$ для исследования магнитоупругих и магнитотепловых свойств вдоль направления оси $c([0001])$ и оси $b([10 \overline{1} 0])$. 
Гидрирование образца проводилось на установке типа Сивертса в условиях низкого давления (до $0.1 \mathrm{MPa}$ ), чтобы избежать разрушения монокристалла. Количество абсорбированного водорода определялось путем измерения разницы давления в камере до и после завершения реакции. Точность определения концентрации водорода составляло 0.01 атома водорода на формульную единицу (at. H/f.u.). Полученный состав $\mathrm{GdH}_{0} .15$ соответствовал твердому раствору металл-водород ( $\alpha$-фаза) $[13,14]$.

В данной работе магнитокалорический эффект (адиабатическое изменение температуры) измерялся прямым методом с использованием установки MagEq MMS 901 (ООО „ПМТиК“, Троицк) в температурном интервале $80-350 \mathrm{~K}$ и в магнитных полях до $1.8 \mathrm{~T}$. Магнитострикция изучалась тензометрическим методом в том же диапазоне температур в магнитных полях до $1.2 \mathrm{~T}$.

\section{3. Результаты и обсуждение}

Известно [15], что феноменологическое описание явления магнитострикции, достаточно сложное. Однако для соединений с одной ярковыраженной осью (каким и является монокристалл гадолиния) существует соотношение, полученное Кларком [16]

$$
\begin{aligned}
\lambda= & \lambda_{1}^{\alpha, 0}\left(\beta_{x}^{2}+\beta_{y}^{2}\right)+\lambda_{2}^{\alpha, 0} \beta_{z}^{2}+\lambda_{1}^{\alpha, 2}\left(\beta_{x}^{2}+\beta_{y}^{2}\right)\left(\alpha_{z}^{2}-1 / 3\right) \\
& +\lambda_{2}^{\alpha, 2} \beta_{z}^{2}\left(\alpha_{z}^{2}-1 / 3\right)+\lambda^{\gamma, 2}\left\{1 / 2\left(\beta_{x}^{2}-\beta_{y}^{2}\right)\left(\alpha_{x}^{2}-\alpha_{y}^{2}\right)\right. \\
& \left.+2 \beta_{x} \beta_{y} \alpha_{x} \alpha_{y}\right\}+\lambda^{\varepsilon, 2}\left(\beta_{x} \alpha_{x}+\beta_{y} \alpha_{y}\right) \beta_{z} \alpha_{z}
\end{aligned}
$$

где $\beta_{i}$ и $\alpha_{I}-$ косинусы углов между направлением измерения магнитострикции и направлением вектора намагниченности относительно осей $x, y$ и $z$ (причем $z \| c$ ). Два первых члена не зависят от направления вектора $\mathbf{M}_{s}$ и описывают изменение размеров кристалла только при изменении $\mathbf{M}_{s}$ по модулю (к примеру, в области парапроцесса). В этом случае $\lambda_{1}^{\alpha, 0}$ определяет магнитострикцию в базисной плоскости кристалла, а $\lambda_{2}^{\alpha, 0}-$ в направлении оси $c$. Остальные члены в выражении (1) характеризуют анизотропную магнитострикцию $\left(\lambda_{1}^{\alpha, 2}, \lambda_{2}^{\alpha, 2}, \lambda^{\gamma, 2}, \lambda^{\varepsilon, 2}\right)$, связанную с изменением ориентации вектора $\mathbf{M}_{s}$.

Нами в результате измерений магнитострикции был получен набор изотерм $\lambda_{i j}(H)$, измеренных вдоль оси $c$ или вдоль оси $b$ монокристалла гадолиния (на это указывает первый индекс $i$ ) для случая, когда внешнее магнитное поле было приложено вдоль оси с или вдоль оси $b$ (см. второй индекс $j$ ). На основе этих измерений нами были построены температурные зависимости $\lambda_{c c}(T), \lambda_{c b}(T), \lambda_{b c}(T), \lambda_{b b}(T)$.

Магнитострикционные константы $\lambda_{1}^{\alpha, 0}, \lambda_{2}^{\alpha, 0}, \lambda_{1}^{\alpha, 2}, \lambda_{2}^{\alpha, 2}$ связаны с полученными из эксперимента $\lambda_{c c}, \lambda_{c b}, \lambda_{b c}$,

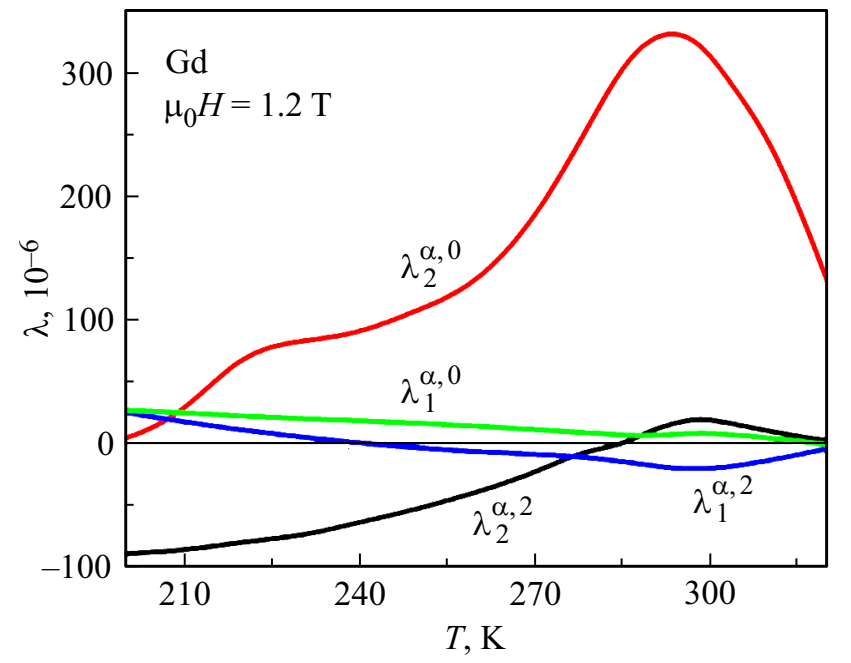

Рис. 1. Температурная зависимость магнитострикционных констант для монокристалла Gd.

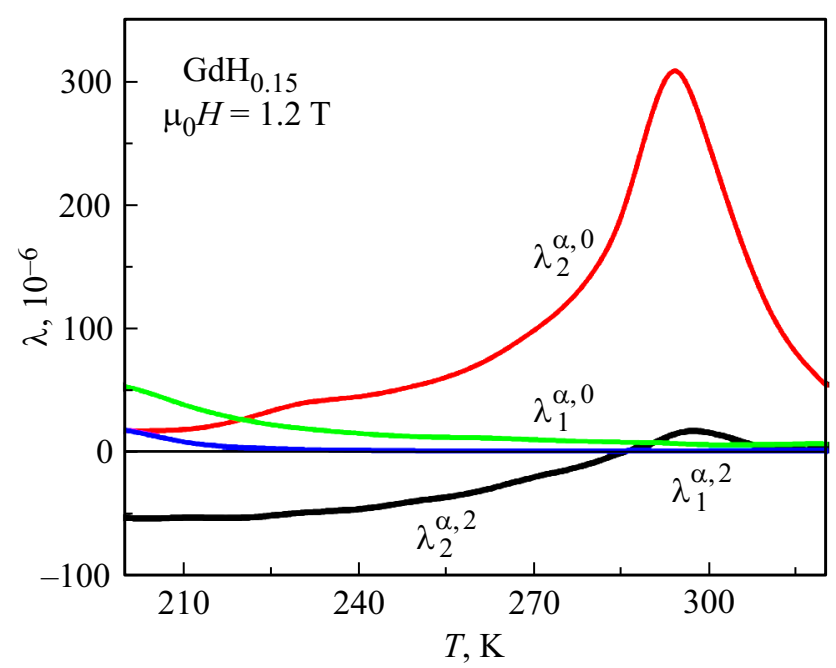

Рис. 2. Температурная зависимость магнитострикционных констант для монокристалла $\mathrm{GdH}_{0.15}$.

$\lambda_{b b}$ следующими соотношениями:

$$
\begin{aligned}
& \lambda_{1}^{\alpha, 0}=\left(2 \lambda_{b b}+\lambda_{b b}\right) / 3, \\
& \lambda_{2}^{\alpha, 0}=\left(2 \lambda_{b c}+\lambda_{c c}\right) / 3, \\
& \lambda_{1}^{\alpha, 2}=\lambda_{c b}-\lambda_{b b}, \\
& \lambda_{2}^{\alpha, 2}=\lambda_{c c}-\lambda_{b c} .
\end{aligned}
$$

На рис. 1 и 2 показаны температурные зависимости магнитострикционных констант $\lambda_{1}^{\alpha, 0}(T), \lambda_{2}^{\alpha, 0}(T), \lambda_{1}^{\alpha, 2}(T)$, $\lambda_{2}^{\alpha, 2}(T)$ для монокристалла $\mathrm{Gd}$ до и после гидрирования в магнитном поле $1.2 \mathrm{~T}$ в интервале температур, охватывающем область обоих магнитных переходов. Следует отметить, что данные для исходного образца согласуются с известными литературными данными $[17,18]$. 
Сравнивая кривые на рис. 1 и 2 можно выделить следующие особенности их поведения. Константа $\lambda_{2}^{\alpha, 0}$, как уже отмечалось выше, определяющая магнитострикцию в направлении оси $c$, демонстрирует большие положительные значения как для исходного, так и для гидрированного образца в области магнитных фазовых переходов, достигая максимума при температуре Кюри. Подобный эффект имеет место и в соединениях на основе РЗМ $[19,20]$. Для исходного $\mathrm{Gd}\left(\lambda_{2}^{\alpha, 0}\right)_{\max }=330 \cdot 10^{-6}$, в то время как для гидрированного образца $\mathrm{GdH}_{0.15}$ $\left(\lambda_{2}^{\alpha, 0}\right)_{\max }$ чуть меньше и равна $310 \cdot 10^{-6}$. Гидрирование значительно уменьшает интервал температур, в котором наблюдаются высокие значение константы. Так, к примеру, интервал температур, в котором $\lambda_{2}^{\alpha, 0}>200 \cdot 10^{-6}$ у $\mathrm{GdH}_{0.15}$ в 2 раза меньше, чем у образца гадолиния без водорода. В результате такого быстрого уменьшения магнитострикционного эффекта с изменением температуры в водородсодержащем образце величина скачка $\lambda_{2}^{\alpha, 0}$ в области СПП меньше в 4 раза, по сравнению c исходным Gd. Сам переход сдвигается в сторону комнатной температуры на $10 \mathrm{~K}$. Константы $\lambda_{1}^{\alpha, 0}$ и $\lambda_{1}^{\alpha, 2}$ в наводороженном образце $\mathrm{GdH}_{0.15}$ также положительные и быстро спадают до 0 при приближении к $T_{\mathrm{C}}$. Исходный образец при этом демонстрирует смену знака константы $\lambda_{1}^{\alpha, 2}$ в области СПП с плюса на минус и широкий размытый пик в области температуры Кюри. Таким образом, нами установлено, что гидрирование может оказывать влияние не только на величину, но и на знак констант магнитострикции даже при небольших концентрациях водорода в образцах.

Важно было также установить, как гидрирование влияет на магнитокалорический эффект в монокристалле $\mathrm{Gd}$, поскольку именно это свойство гадолиния нашло столь широкое применение на практике. На рис. 3 показана зависимость магнитокалорического эффекта от температуры в области магнитных фазовых переходов в монокристалле $\mathrm{GdH}_{0.15}$ при изменении внешнего магнитного поля на величину $\mu_{0} \Delta H=0.5,0.8,1.2,1.8 \mathrm{~T}$ и для сравнения - в монокристалле $\mathrm{Gd}$ при $\mu_{0} \Delta H=1.8 \mathrm{~T}$. Магнитное поле было приложено вдоль оси $c$ (рис. $3, a$ ) и вдоль оси $b$ (рис. $3, b)$. В обоих составах скачков МКЭ в области СПП не наблюдалось. Однако было обнаружено, что в области температуры Кюри МКЭ в водородосодержащем образце $\mathrm{Gd}$ сохраняет анизотропный характер даже в максимально доступном для нас магнитном поле $1.8 \mathrm{~T}$ ( $5 \mathrm{~K}$ при приложении поля вдоль оси $b$ и $4.2 \mathrm{~K}-$ вдоль оси $c$ ), в то время как исходный $\mathrm{Gd}$ в данном поле демонстрирует практически изотропный характер, что хорошо согласуется с известными литературными данными [6].

Наблюдаемые нами явления можно объяснить, если принять во внимание результаты исследования, выполненного для других РЗМ $[21,22]$. Было установлено, что размещение атомов водорода в решетке редкоземельных металлов не является беспорядочным даже при их малом содержании. Атомы водорода, занимая определенные позиции, способны создавать упорядоченную
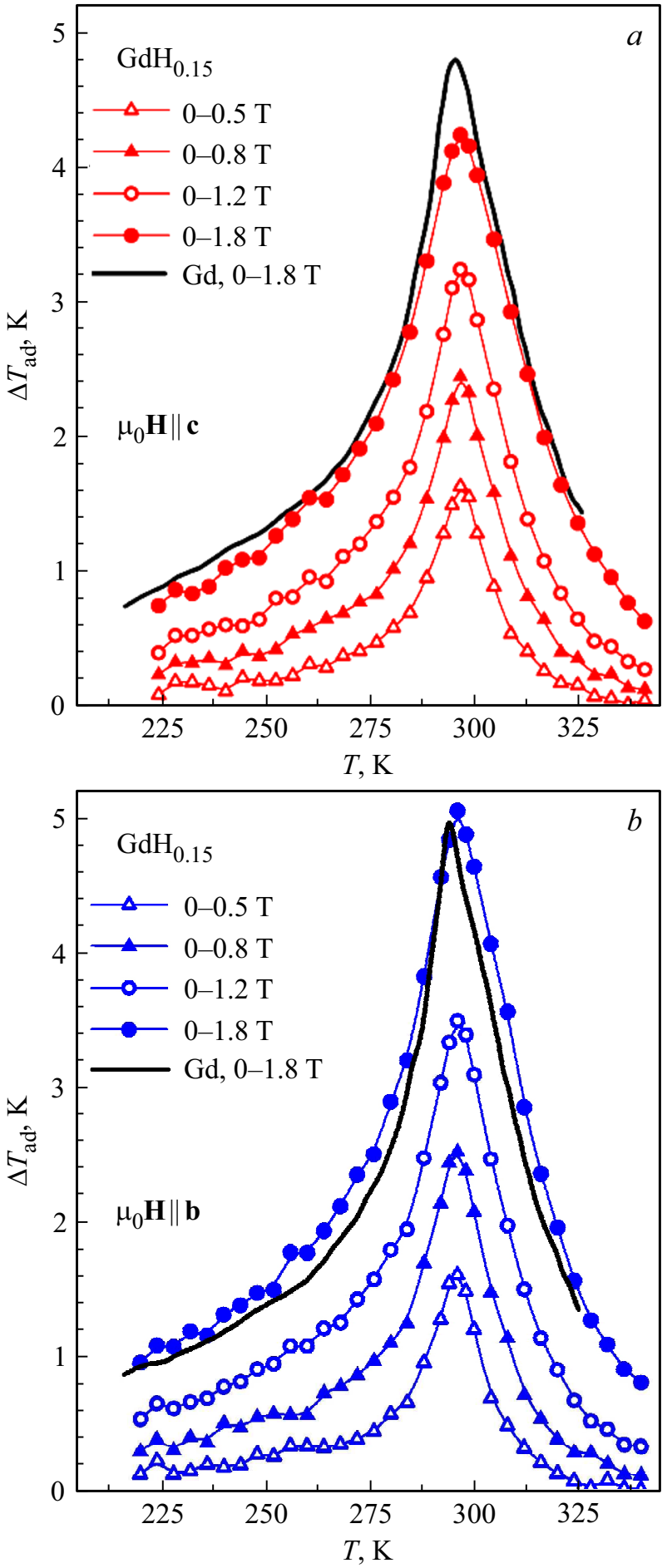

Рис. 3. Магнитокалорический эффект в монокристалле $\mathrm{GdH}_{0.15}$ при $\mu_{0} \Delta H=0.5,0.8,1.2,1.8$ Т и в монокристалле $\mathrm{Gd}$ при $\mu_{0} \Delta H=1.8 \mathrm{~T}$. Магнитное поле вдоль оси $c(a)$ и вдоль оси $b(b)$.

структуру (происходит выстраивание атомов водорода в цепочки вдоль оси $c$, возникновению локальной анизотропии $[23,24])$. Такое упорядочение было подтверждено с помощью нейтронографических исследований в монокристалле $\alpha-\mathrm{LuD}_{x}$ [25]. Именно это явление, в свою 
очередь, и может приводить к возникновению индуцированной водородом анизотропии магнитокалорического эффекта.

\section{4. Заключение}

Исследования, проведенные на монокристаллических образцах $\mathrm{Gd}$ и его твердого раствора внедрения $\mathrm{GdH}_{0.15}$, позволили нам продемонстрировать влияние гидрирования на магнитострикционные и магнитокалорические свойства гадолиния. При этом заметное влияние на магнитострикцию обнаружено как в области перехода „порядок“-,порядок“, так и в области перехода „порядок“-,беспорядок“, а влияние водорода, внедренного в кристаллическую решетку гадолиния, на МКЭ наиболее ярко выражено в области перехода „порядок“-,беспорядок“. Наблюдаемые эффекты могут быть связаны с появлением локальной анизотропии, возникающей в образцах вследствие упорядочения водорода в кристаллической решетке Gd.

Авторы выражают благодарность В. Vondráčková и J. Pospíšil за получение исходных образцов гадолиния.

\section{Список литературы}

[1] С.А. Никитин. Магнитные свойства редкоземельных металлов и их сплавов. Из-во МГУ, М. (1989). 247 с.

[2] К.П. Белов. Магнитострикционные явления и их приложения. Наука, М. (1987). 240 с.

[3] A.M. Tishin, Y.I. Spichkin. The magnetocaloric effect and its application. Institute of Physics Publishing. Bristol, Philadelphia (2003). 480 p.

[4] A. Kitanovski, J. Turek, U. Tomc, U. Plaznik, M. Ožbolt, A. Poredoš. Magnetocaloric Energy Conversion From Theory to Applications. Cham: Springer International Publishing Switzerland (2015). $456 \mathrm{p}$.

[5] S.Y. Dan'kov, A.M. Tishin, V.K. Pecharsky, K.A. Gschneidner. Phys. Rev. B 57, 3478 (1998).

[6] A.M. Aliev, A.B. Batdalov, L.N. Khanov, V.V. Koledov, V.G. Shavrov, I.S. Tereshina, S.V. Taskaev. J. Alloys Comp. 676, 601 (2016).

[7] A.P. Kamantsev, V.V. Koledov, V.G. Shavrov, I.S. Tereshina. Solid State Phenomena 215, 113 (2014).

[8] G.S. Burkhanov, N.B. Kolchugina, E.A. Tereshina, I.S. Tereshina, G.A. Politova, V.B. Chzhan, D. Badurski, O.D. Chistyakov, M. Paukov, H. Drulis, L. Havela. Appl. Phys. Lett. 104, 242402 (2014)

[9] E.A. Tereshina, S. Khmelevskyi, G. Politova, T. Kaminskaya, H. Drulis, I.S. Tereshina. Sci. Rep. 6, 22553 (2016).

[10] И.С. Терешина, В.Б. Чжан. ФТТ 60, 1103 (2018).

[11] А.Н. Четырбоцкий, И.С. Терешина, В.А. Четырбоцкий, Физикохимия поверхности и защита материалов 54, 201 (2018).

[12] V. Chzhan, I. Tereshina, G. Burkhanov, G. Politova, H. Drulis. EPJ Web of Conferences, 185, 05011 (2018).

[13] P. Vajda. J. Alloys Compd. 404-406, 131 (2005).
[14] H. Yayama, A. Tomokiyo. Advances in Cryogenic Engineering (Materials) / Eds F.R. Fickett, R.P. Reed, Plenum Press, N.Y. 38, 293 (1992).

[15] С.В. Вонсовский, Я.С. Щур. Ферромагнетизм. Наука, М. (1965).

[16] A.E. Clark, B.F. Desavage, R. Bozorth. Phys. Rev. A 138, 216 (1965).

[17] К.П. Белов, М.А. Белянчикова, Р.З. Левитин, С.А. Никитин. Редкоземельные ферро и антиферромагнетики. Наука, M. (1965). 319 c.

[18] R.M. Bozorth, T. Wakiama. J. Phys. Soc. Jpn 18, 97 (1963).

[19] С.А. Никитин, И.С. Терешина, Н.Ю. Панкратов, Е.А. Терешина, Ю.В. Скурский, К.П. Скоков, Ю.Г. Пастушенков. ФTT 43, 1651 (2001).

[20] I. Tereshina, G. Politova, E. Tereshina, S. Nikitin, G. Burkhanov, O. Chistyakov, A. Karpenkov. J. Phys. Conf. Ser. 200, 092012 (2010).

[21] P. Vajda, J.N. Daou. J. Less-Common Met. 101, 269 (1984).

[22] P. Vajda. Handbook on the Physics and Chemistry of Rare Earths / Eds K.A. Gschneidner,Jr., Le Roy Eyring. NorthHolland, Amsterdam (1995). V. 20. P. 207.

[23] S. Nikitin, I. Tereshina, E. Tereshina, W. Suski, H. Drulis. J. Alloys Comp. 451, 477 (2008).

[24] E.A. Tereshina, H. Drulis, Y. Skourski, I. Tereshina. Phys. Rev. B 87, 214425 (2013).

[25] O. Blaschko, G. Krexner, J.N. Daou, P. Vajda. Phys. Rev. Lett. 55, 2876 (1985).

Редактор Ю.Э. Китаев 